\title{
Quasiparticle Tunneling across an Exciton Condensate
}

\author{
Ding Zhang ${ }^{1,2,3,{ }^{*}}$ Joseph Falson ${ }^{2, \S}$ Stefan Schmult, ${ }^{2, \dagger}$ Werner Dietsche, ${ }^{2}$ and Jurgen H. Smet ${ }^{2, \$}$ \\ ${ }^{1}$ State Key Laboratory of Low-Dimensional Quantum Physics and Department of Physics, Tsinghua University, Beijing 100084, China \\ ${ }^{2}$ Max Planck Institute for Solid State Research, Heisenbergstrasse 1, D-70569 Stuttgart, Germany \\ ${ }^{3}$ Beijing Academy of Quantum Information Sciences, Beijing 100193, China
}

(Received 7 August 2019; revised manuscript received 2 January 2020; accepted 3 June 2020; published 19 June 2020)

\begin{abstract}
The bulk properties of the bilayer quantum Hall state at total filling factor one have been intensively studied in experiment. Correlation induced phenomena such as Josephson-like tunneling and zero Hall resistance have been reported. In contrast, the edge of this bilayer state remains largely unexplored. Here, we address this edge physics by realizing quasiparticle tunneling across a quantum point contact. The tunneling manifests itself as a zero bias peak that grows with decreasing temperature. Its shape agrees quantitatively with the formula for weak quasiparticle tunneling frequently deployed in the fractional quantum Hall regime in single layer systems, consistent with theory. Interestingly, we extract a fractional charge of only a few percent of the free electron charge, which may be a signature of the theoretically predicted leakage between the chiral edge and the bulk mediated by gapless excitations.
\end{abstract}

DOI: 10.1103/PhysRevLett.124.246801

The $\nu_{\text {tot }}=1 / 2+1 / 2$ quantum Hall state (QHS) [1] is an archetypical multicomponent state described by Halperin's $(1,1,1)$ wave function [2] that keeps attracting attention [3-8]. It was first realized in GaAs double quantum well systems $[9,10]$ and more recently in graphene double bilayers [11-13]. This ground state of the system can be well understood as a condensate of excitons $[1,14]$. The formation of such a condensate breaks the U(1) symmetry of the system [15], giving rise to gapless excitations, i.e., Goldstone modes in the bulk. In the pseudospin language, such an excitation corresponds to the generation of a quasiparticle referred to as a meron that can be viewed as half a skyrmion [16,17]. A meron carries an electrical charge of $\pm(e / 2)$ but tends to bind a second meron with opposite vorticity. Immediately following the early excitement on the quantum Hall bilayer experiments [18], Lopez and Fradkin [19] constructed an effective field theory for the edge states. They showed that the edge in a $(m, m, m)$ Halperin multicomponent QHS is largely analogous to that of the 1/ $m$ Laughlin QHS itself ( $m$ is an integer), but there exists leakage from the edge to the bulk due to the presence of gapless excitations [20] [inset of Fig. 1(a)]. It was proposed that an interedge tunneling experiment should be carried out to verify the theory and shed light on the edgebulk interactions [19].

Published by the American Physical Society under the terms of the Creative Commons Attribution 4.0 International license. Further distribution of this work must maintain attribution to the author(s) and the published article's title, journal citation, and DOI. Open access publication funded by the Max Planck Society.
In vivid contrast to the extensive and fruitful experimental studies of the bulk properties of quantum Hall bilayer systems [4,18,21-26], the edge physics has remained unexplored for nearly two decades. While these studies were for a long time the exclusive privilege of the GaAs community, by now an abundance of multicomponent states has been unveiled in a number of other material systems such as graphene based van der Waals heterostructures as well as topological insulators [6,11-13,27-30]. Theoretical insights have suggested that non-Abelian properties of excitations can be harnessed in quantum Hall bilayers by manipulating its edge [31-35]. All these developments make a disclosure of edge physics in bilayers even more imperative. The need for both global top and back gates in quantum Hall bilayers certainly hampered progress, as there was little room for introducing extra gates to manipulate the edge. With the advent of lowdensity $\left(<2 \times 10^{10} \mathrm{~cm}^{-2}\right) \mathrm{GaAs}$ bilayer samples with high quality $\left(\mu \geq 1 \times 10^{6} \mathrm{~cm}^{2} / \mathrm{Vs}\right)$, it has become possible to enter the $\nu_{\text {tot }}=1$ state by only tuning a back gate [36], leaving the top surface available to deploy the split gate technique for edge manipulation. The latter has been widely used to address interedge tunneling for instance in single layer electron systems at fractional filling factors with both odd [37] and even [38-42] denominators. These studies extracted effective charges that are overall close to the theoretical expectations, although the extracted Coulomb interaction strength may be influenced by the device geometry [43].

In this Letter, we successfully realize quasiparticle tunneling in a GaAs bilayer and investigated its edge state for the $\nu_{\text {tot }}=1$ QHS. We obtain a clear zero bias peak in the tunnel conductance of a quantum point contact (QPC). 

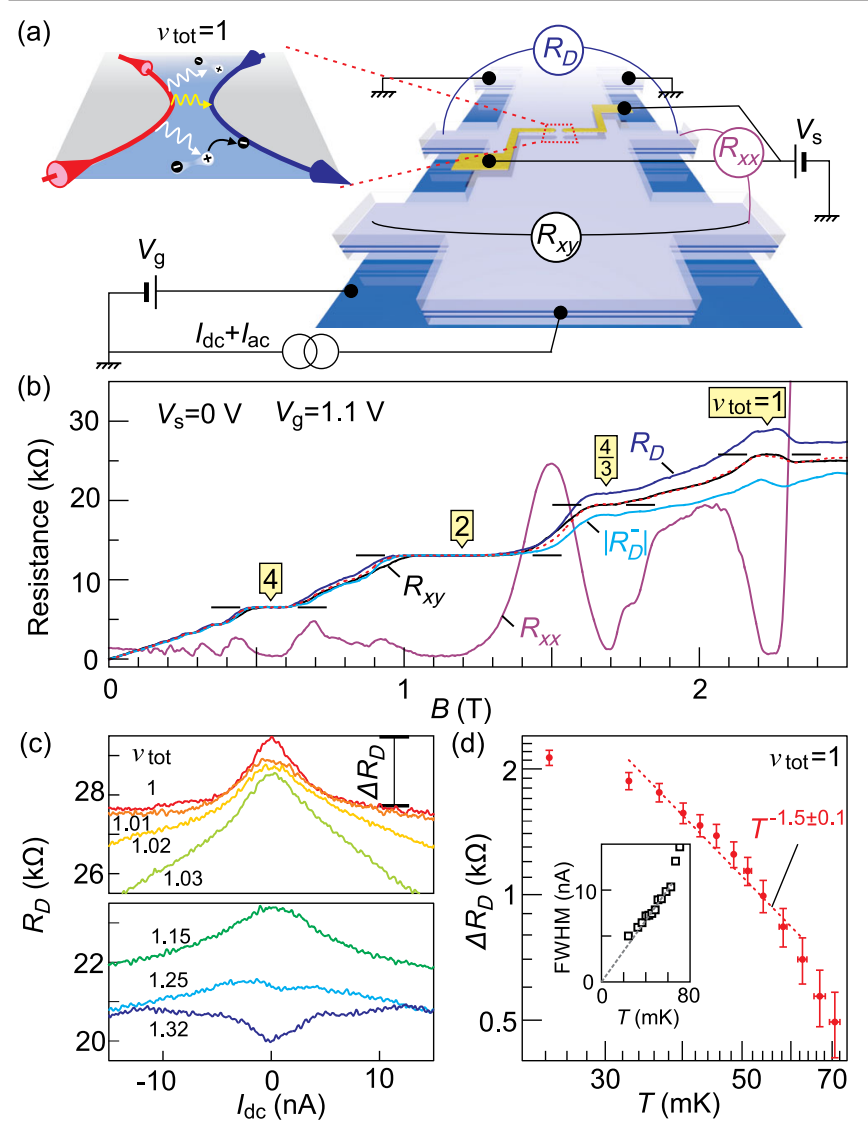

FIG. 1. (a) Schematic illustration of the quantum Hall bilayer device and the measurement configuration. The upper-left inset sketches the quasiparticle tunneling between two counterpropagating edges of the $\nu_{\text {tot }}=1$ state. (b) Hall, longitudinal, and diagonal resistances as a function of magnetic field for the GaAs bilayer in the balanced case measured at the base temperature of the dilution refrigerator. The dotted curve is the calculated average $\left(R_{D}+\left|R_{D}^{-}\right|\right) / 2$. (c) Diagonal resistance $R_{D}$ as a function of dc bias current at different magnetic fields (filling factors are indicated). (d) $\Delta R_{D}$ (see text) as a function of temperature. The dotted line is a fit to the data points. The inset shows the temperature dependence of the full width at half maximum (FWHM) of the tunneling peak.

The peak and its temperature dependence fit well to the theoretical formula derived for weak quasiparticle tunneling in a single layer [44], confirming the theoretically predicted correspondence between the bilayer QHS and the single layer states [19]. Interestingly, the extracted quasiparticle charge is only a few percent of the free electron charge, presumably reflecting a distinct type of interaction between the quasiparticle on the edge and the excitonic condensate in the bulk [20]. The presence of gapless excitations in the bulk [15] allows for leakage from the edge [19] and this process should be taken into account for a complete theory of quasiparticle tunneling [44] in bilayers.

The bilayers in this study reside in a double quantum well of a GaAs $/ \mathrm{Al}_{x} \mathrm{Ga}_{1-x}$ As heterostructure grown by molecular beam epitaxy [36]. Split gates made out of Ti/Au were fabricated on top of the surface with the help of electronbeam lithography, thermal evaporation and a lift-off procedure [45]. The sample was measured in two ${ }^{3} \mathrm{He}-{ }^{4} \mathrm{He}$ dilution refrigerators with a base temperature of about $20 \mathrm{mK}$. The total density - tuned by applying a back gate voltage $\left(V_{g}\right)$-ranged from $4.5-6.0 \times 10^{10} \mathrm{~cm}^{-2}$ with the balanced case corresponding to a density of $5.4 \times 10^{10} \mathrm{~cm}^{-2}$. The mobility exceeds $1.1 \times 10^{6} \mathrm{~cm}^{2} / \mathrm{V}$ s. Resistances were measured using heterodyne detection for an ac current excitation between 0.2 and $0.5 \mathrm{nA}$ at a frequency of 11.3 to $13.3 \mathrm{~Hz}$.

Figure 1(b) displays the resistance curves in the balanced case by using the measurement configuration shown in Fig. 1(a). $R_{x x}$ and $R_{x y}$ reflect the transport in the bulk region. They show well-defined sequences of QHS for such a balanced bilayer. In comparison, $R_{D}$ and $R_{D}^{-}$are the diagonal resistances measured across the QPC for positive and negative magnetic fields, respectively. $R_{D}$ and $\left|R_{D}^{-}\right|$ significantly deviate from $R_{x y}$ at filling factors below 2 , even if no voltage is applied to the split gate $\left(V_{s}=0\right)$. It indicates that the QPC is already defined due to a work function difference between the metallic gate material and GaAs causing significant depletion of electrons in the bilayer system. Measurements on a GaAs bilayer from the same wafer with a global top gate confirm that the metallic gate reduces the electron density by about 2 to $3 \times 10^{10} \mathrm{~cm}^{-2}$ and the regions beneath the metallic gates with a density of about $2 \times 10^{10} \mathrm{~cm}^{-2}$ become well insulating at magnetic fields exceeding $2 \mathrm{~T}$. Current is then forced to flow through the narrow constriction area only and some current may get diverted to the other side of the QPC. Even if so, the $R_{D}(B)$ and $\left|R_{D}^{-}(-B)\right|$ curves still exhibit the plateaus associated with the integer quantum Hall effect at the same magnetic fields as those of the bulk [see Fig. 1(b)]. This overlap suggests that the density in the QPC itself is close to the bulk value-a prerequisite for weak quasiparticle tunneling. The deviation of $R_{D}$ from $R_{x y}$ at $\nu_{\text {tot }}<2$ reflects quasiparticle tunneling between the two counterpropagating edges. According to the LandauerBüttiker formalism [46], this interpretation would give rise to an exact overlap between the calculated average (dotted line) of the diagonal resistances, $\left(R_{D}+\left|R_{D}^{-}\right|\right) / 2$, and $R_{x y}$. This is indeed the case, in particular also at the total filling factor 1 , i.e., $\nu_{\text {tot }}=1 / 2+1 / 2$.

The successful implementation of quasi-particle tunneling in a bilayer at $\nu_{\text {tot }}=1$ can also be demonstrated by studying the current and temperature dependence. When applying a dc source-drain current $\left(I_{\mathrm{dc}}\right)$, a Hall potential equal to $R_{x y} I_{\mathrm{dc}}$ builds up between the two counterpropagating edge states. It detunes them energetically and results in a suppression of the quasiparticle tunneling. Since the tunneling conductance is proportional to the diagonal resistance, i.e., $g_{T}=\left(R_{D}-R_{x y}\right) / R_{x y}^{2}$, a suppressed $g_{T}$ at finite $I_{\mathrm{dc}}$ manifests itself as a zero-bias peak in $R_{D}$. Experimentally, we obtain such a zero-bias peak as shown 

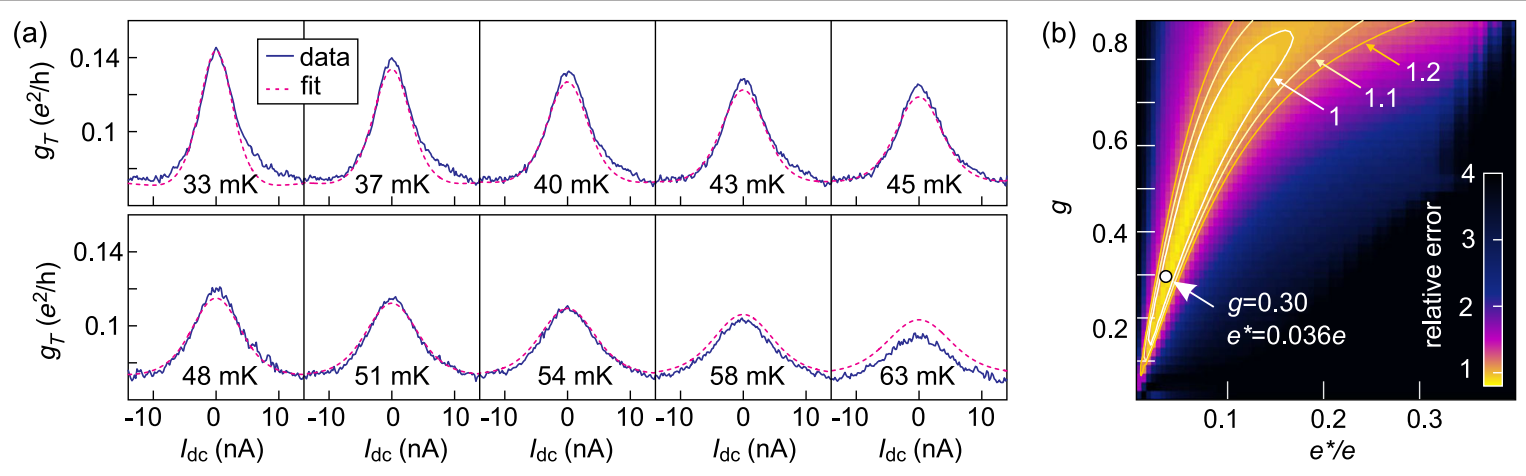

FIG. 2. (a) Tunnel conductance (solid curve) as a function of dc current for ten different temperatures. Also shown are fit curves (dashed lines) generated with a single set of optimized fit parameters obtained by simultaneously fitting the formula for weak quasiparticle tunneling for all traces and minimizing the total error. The fitted $g$ and $e^{*}$ are 0.30 and $0.036 e$. (b) Normalized fit error [48] as a function of $g$ and $e^{*} / e$. The circle marks the best fit point. The contours correspond to relative errors of $1,1.1$, and 1.2 , as indicated by the arrows.

in Fig. 1(c). The peak feature depends sensitively on the magnetic field and appears only around $\nu_{\text {tot }}=1$ [45]. The dip at $\nu_{\text {tot }}=4 / 3$ may stem from weaker interedge coupling [37]. Figure 1(d) addresses the temperature dependence of the peak at $\nu_{\text {tot }}=1$. The peak height $\Delta R_{D}$ is defined as the difference between the maximum value in each $R_{D}-I_{\mathrm{dc}}$ curve and the background resistance [illustrated in Fig. 1(c)]. According to theory, the peak height should exhibit a power-law dependence [44], $T^{2 g-2}$, where $g$ measures the Coulomb interaction strength. We fit our data between 30 and $65 \mathrm{mK}$ and obtain a $T^{-1.5 \pm 0.1}$ dependence. The inset of Fig. 1(d) also demonstrates that the peak width scales linearly with $T$ and approaches zero at $T=0$, as expected from theory [44] and observed in single layer systems $[38,47]$. As the temperature approaches base temperature, $\Delta R_{D}$ starts to saturate. The quasiparticle tunneling possibly enters the strong tunneling regime as the interedge coupling gets enhanced at lower temperatures $[40,47]$. However, given the very low electron density of our sample $\left(<3 \times 10^{10} \mathrm{~cm}^{-2}\right)$ and the relatively high contact resistance $\left(\sim h / e^{2}\right)$, difficulties in reducing the electron temperature in step with the mixing chamber temperature may also play an important role and be responsible for the saturation. We therefore use the data in the range of 30 to $65 \mathrm{mK}$ for the following quantitative analysis.

Since all features of the peak at $\nu_{\text {tot }}=1$ seem to be consistent with the scenario of weak quasiparticle tunneling, we employ the formula derived for single layer systems in the weak-tunneling limit $[38,44]$ to fit our data:

$$
\begin{aligned}
g_{T} & =A T^{2 g-2} F\left(g, \frac{e^{*} I_{D C} R_{x y}}{k_{B} T}\right)+g_{\infty}, \\
F(g, x)= & B\left(g+i \frac{x}{2 \pi}, g-i \frac{x}{2 \pi}\right) \\
& \times\left\{\pi \cosh \left(\frac{x}{2}\right)-2 \sinh \left(\frac{x}{2}\right) \operatorname{Im}\left[\Psi\left(g+i \frac{x}{2 \pi}\right)\right]\right\} .
\end{aligned}
$$

Here, $B(x, y)$ and $\Psi(x)$ are the Euler beta function and the digamma function, respectively. $e^{*}$ represents the fractional charge of the quasiparticles. $g$ is the Coulomb interaction constant. $A$ and $g_{\infty}$ (background conductance) are constants.

Figure 2(a) presents the fits to the experimental data using Eq. (1) when the layers have equal densities. We fit simultaneously the peaks (dotted lines) at ten temperature points with $e^{*}, g, A$, and $g_{\infty}$ as the four fitting parameters. The experimental results can be satisfactorily described by the weak tunneling formula, as expected from the LopezFradkin theory [19]. The extracted interaction constant is $g=0.30$. However, the fitted fractional charge $e^{*}$ is only a small fraction of the electron charge. The smallness of $e^{*}$ can be further appreciated by evaluating the uncertainty of the fitted values. Figure 2(b) displays the relative error, defined as the fit residual normalized by the experimental noise, as has been done previously in single layer systems $[38,40,42]$. A fit error below 1 indicates that the corresponding $g$ and $e^{*}$ values are allowed because the fit is consistent with the data within experimental noise. Figure 2(b) shows that the region with a low fit error is confined well below $e^{*}=0.2 e$, much smaller than $0.5 e$ for a meron or $e$ for a quasiparticle on the edge [19].

We further study the quasiparticle tunneling, if an imbalance exists between the density of the layers. In transport, the $\nu_{\text {tot }}=1$ state is robust against such an imbalance [36], but the nature of the underlying ground states change. When ramping up $V_{g}$ from $0.5 \mathrm{~V}$ to about $1.4 \mathrm{~V}$, the QHS evolves continuously from the single layer $\nu_{t}=1$ state to the $\nu_{\text {tot }}=\nu_{t}+\nu_{b}=1 / 2+1 / 2$ state and finally into the $\nu_{\text {tot }}=1 / 3+2 / 3$ state. Here, $\nu_{t}$ and $\nu_{b}$ refer to the filling factors of the top and bottom layer, respectively. A larger $V_{g}$ also gives rise to a higher density such that the $\nu_{\text {tot }}=1$ state shifts to a higher magnetic field. The effective layer separation, $d / l_{B}$, therefore becomes larger due to the decrease of the magnetic length $l_{B}$ at a fixed layer distance $d$, resulting in a weaker $\nu_{\text {tot }}=1$ state [36]. 


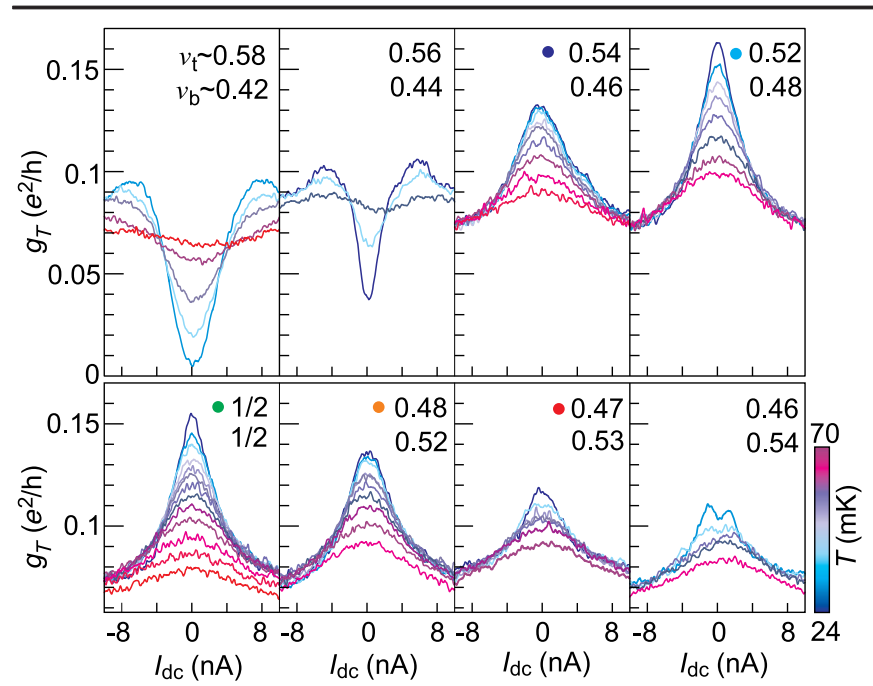

FIG. 3. Tunnel conductance as a function of bias current at different temperatures and for different degrees of imbalance between the layers. $V_{g}$ is varied from $0.9 \mathrm{~V}$ (top leftmost panel) to $1.25 \mathrm{~V}$ (bottom rightmost panel) in $0.05 \mathrm{~V}$ steps. The estimated filling factor in the top $\left(\nu_{t}\right)$ and bottom $\left(\nu_{b}\right)$ layer have been included in each panel. The split gate voltage to form the QPC is kept constant at $V_{s}=0.5 \mathrm{~V}$ after cycling to $-1.5 \mathrm{~V}$ at base temperature.

Drastic changes occur in the tunnel conductance as shown in Fig. 3, even for rather small degrees of imbalance. A deep minimum around zero bias is observed when $V_{g}$ is set to 0.9 and $0.95 \mathrm{~V}$. The tunnel conductance suddenly transforms into a peak at $V_{g}=1 \mathrm{~V}$. The background conductance $g_{\infty}$, which serves as a measure of the transmission through the QPC, stays almost the same across this gate voltage interval. This is very different from previous experiments [37,47] showing dip-to-peak transitions. We assign this observed behaviour to a crossover from the breakdown of the quantum Hall effect to interedge tunneling. This is supported by the fact that for $V_{g}=0.9 \mathrm{~V}, g_{T}$ is almost zero at $I_{\mathrm{dc}}=0$, suggesting near perfect transmission through the QPC. Although the split gate voltage $V_{s}$ is left unchanged for different $V_{g}$, the range of filling factor where the incompressible $\nu_{\text {tot }}=1$ state forms, shrinks as $V_{g}$ increases from 0.9 to $1.1 \mathrm{~V}$. This is manifested by a narrowing of the quantum Hall plateau at $\nu_{\text {tot }}=1$ $[36,45]$. It effectively results in a reduction of the spatial distance between the two edges. By going to higher $V_{g}$ such that $\nu_{b}>\nu_{t}$, the peak height decreases because the $\nu_{\text {tot }}=1$ state becomes weaker at a higher total density [36].

In Fig. 4 we explore the influence of the small imbalance among the two layers on the parameters $g$ and $e^{*} / e$ required to fit the quasiparticle tunnel conductance peak when varying $V_{g}$ near the balanced point between $V_{g}=1.0$ and $1.2 \mathrm{~V}$. We consistently get a small charge of $0.1 e$ or even smaller. Similar results were obtained either from the same QPC upon different thermal cycling or from another QPC [45]. This reproducibility rules out the scenario of

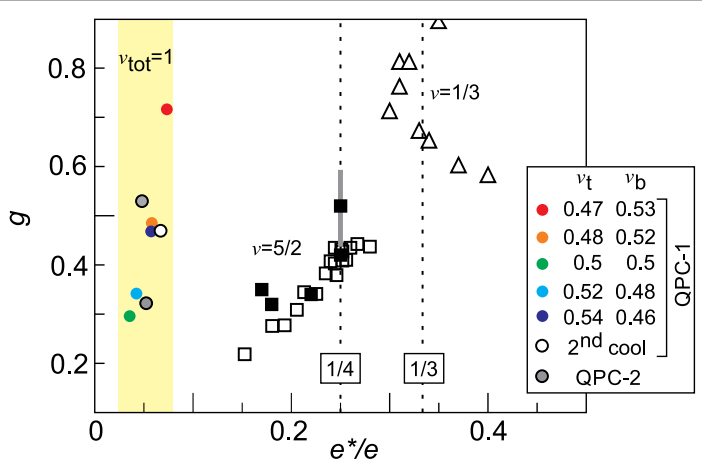

FIG. 4. Fitted values of $g$ and $e^{*} / e$ for the $\nu_{\text {tot }}=1$ state near the balance point. Data points for $\nu=5 / 2$ from Refs. [38,40-42] and for $\nu=1 / 3$ from Ref. [37] are also shown. The error bar for one of the $\nu=5 / 2$ data points stems from a variation of the confinement while fixing $e^{*} / e=1 / 4$ during fitting [42]. Empty squares for $\nu=5 / 2$ are extracted by varying the QPC gate voltage [41] at a fixed magnetic field (we avoid taking the points in the accidental resonant situation). Vertical dotted lines at $1 / 4$ and $1 / 3$ correspond to the expected ratio of $e^{*} / e$ for the $5 / 2$ and $1 / 3 \mathrm{FQH}$ states, respectively.

incidental resonant tunneling with local charges in the QPC as the mechanism for the reduced $e^{*}[41]$. We also note that the influence from the neighboring FQHS should be minimal in the balanced situation as the individual layer is at $\nu=1 / 2$. Figure 4 also includes previous experimental results gathered on single layer systems at $\nu=5 / 2$ [38,40-42] and $\nu=1 / 3$ [37] with the same approach. Analogously, for those works the extracted $g$ spans a wide range of values and exhibits a strong dependence on the transmission through the QPC $[37,41,42]$, possibly via the device geometry effect [43]. However, the extracted $e^{*} / e$ values in previous experiments all crowd around the theoretically expected values (vertical dotted lines). The variation in the Coulomb interaction due to different confinement potential almost exclusively affects the estimated $g$, whereas $e^{*}$ apparently reflects an intrinsic property of the quasiparticle. The small fractional charge in our bilayer cannot be simply explained by the geometry effect.

Instead, it may reveal the underlying interaction between the edge and the bulk of the bilayer. We recall that Eq. (1) was derived for tunneling between chiral Luttinger liquids across a gapped bulk [44]. In contrast, the $\nu_{\text {tot }}=1$ state possesses gapless excitations in the bulk [15], which offers an additional channel for the chiral edge to interact with. This is referred to as an "open Luttinger liquid" [20]. Lopez and Fradkin have argued that this blurs the distinction between the edge and the bulk. Excitations on the edge can directly leak into the bulk. The same may occur in the opposite direction on the other side and tunneling across the bulk in the usual sense would not be required [19]. This process is schematically depicted in Fig. 1(a) and this process would be nonlocal [19]. We speculate that the 
leakage effectively reduces the amount of charge that reaches the other edge [49].

In summary, interedge quasiparticle tunneling in a quantum Hall bilayer was finally realized experimentally. We observed the tunneling peak at the $\nu_{\text {tot }}=1$ QHS. The extracted fractional charge amounts to an unusually small value-only a few percent of the electron charge, which may be caused by the leakage from the edge to the bulk via the gapless excitations.

We thank Klaus von Klitzing, Hailong Fu, Pengjie Wang, Haiwen Liu, Xincheng Xie, and Timo Hyart for fruitful discussions. We thank Marion Hagel for technical assistance. Self-consistent simulations of the quantum point contact were carried out with the nextnano software package. This study was financially supported by the National Natural Science Foundation of China (Grants No. 11922409, No. 11790311, and No. 11604176) and the German Ministry of Education and Research (BMBF Grant No. 01BM900).

*dingzhang@mail.tsinghua.edu.cn

${ }^{\dagger}$ Present address: TU Dresden, Electrical and Computer Engineering, Institute of Semiconductors and Microsystems, Nöthnitzer Str. 64, 011187 Dresden, Germany.

*j.smet@fkf.mpg.de

${ }^{\S}$ Present address: Department of Applied Physics and Materials Science, California Institute of Technology, Pasadena, CA, USA.

[1] J. Eisenstein, Annu. Rev. Condens. Matter Phys. 5, 159 (2014).

[2] D. Yoshioka, A. H. MacDonald, and S. M. Girvin, Phys. Rev. B 39, 1932 (1989).

[3] L. Tiemann, W. Wegscheider, and M. Hauser, Phys. Rev. Lett. 114, 176804 (2015).

[4] D. Zhang, W. Dietsche, and K. von Klitzing, Phys. Rev. Lett. 116, 186801 (2016).

[5] Z. Zhu, L. Fu, and D. N. Sheng, Phys. Rev. Lett. 119, 177601 (2017).

[6] D. V. Fil and S. I. Shevchenko, Low Temp. Phys. 44, 867 (2018).

[7] B. Lian and S.-C. Zhang, Phys. Rev. Lett. 120, 077601 (2018).

[8] J. P. Eisenstein, L. N. Pfeiffer, and K. W. West, Phys. Rev. Lett. 123, 066802 (2019).

[9] Y. W. Suen, L. W. Engel, M. B. Santos, M. Shayegan, and D. C. Tsui, Phys. Rev. Lett. 68, 1379 (1992).

[10] J. P. Eisenstein, G. S. Boebinger, L. N. Pfeiffer, K. W. West, and S. He, Phys. Rev. Lett. 68, 1383 (1992).

[11] X. Liu, K. Watanabe, T. Taniguchi, B. I. Halperin, and P. Kim, Nat. Phys. 13, 746 (2017).

[12] J. Li, T. Taniguchi, K. Watanabe, J. Hone, and C. R. Dean, Nat. Phys. 13, 751 (2017).

[13] G. W. Burg, N. Prasad, K. Kim, T. Taniguchi, K. Watanabe, A. H. MacDonald, L. F. Register, and E. Tutuc, Phys. Rev. Lett. 120, 177702 (2018).
[14] J. P. Eisenstein and A. H. MacDonald, Nature (London) 432, 691 (2004).

[15] I. B. Spielman, J. P. Eisenstein, L. N. Pfeiffer, and K. W. West, Phys. Rev. Lett. 87, 036803 (2001).

[16] K. Moon, H. Mori, K. Yang, S. M. Girvin, A. H. MacDonald, L. Zheng, D. Yoshioka, and S.-C. Zhang, Phys. Rev. B 51, 5138 (1995).

[17] K. Yang, K. Moon, L. Belkhir, H. Mori, S. M. Girvin, A. H. MacDonald, L. Zheng, and D. Yoshioka, Phys. Rev. B 54, 11644 (1996).

[18] I. B. Spielman, J. P. Eisenstein, L. N. Pfeiffer, and K. W. West, Phys. Rev. Lett. 84, 5808 (2000).

[19] A. Lopez and E. Fradkin, Phys. Rev. B 63, 085306 (2001).

[20] A. H. Castro Neto, C. de C. Chamon, and C. Nayak, Phys. Rev. Lett. 79, 4629 (1997).

[21] M. Kellogg, I. B. Spielman, J. P. Eisenstein, L. N. Pfeiffer, and K. W. West, Phys. Rev. Lett. 88, 126804 (2002).

[22] E. Tutuc, M. Shayegan, and D. A. Huse, Phys. Rev. Lett. 93, 036802 (2004).

[23] M. Kellogg, J. P. Eisenstein, L. N. Pfeiffer, and K. W. West, Phys. Rev. Lett. 93, 036801 (2004).

[24] L. Tiemann, W. Dietsche, M. Hauser, and K. von Klitzing, New J. Phys. 10, 045018 (2008).

[25] X. Huang, W. Dietsche, M. Hauser, and K. von Klitzing, Phys. Rev. Lett. 109, 156802 (2012).

[26] D. Nandi, A. D. K. Finck, J. P. Eisenstein, L. N. Pfeiffer, and K. W. West, Nature (London) 488, 481 (2012).

[27] J. Su and A. H. MacDonald, Nat. Phys. 4, 799 (2008).

[28] S. Tran, J. Yang, N. Gillgren, T. Espiritu, Y. Shi, K. Watanabe, T. Taniguchi, S. Moon, M. Baek, D. Smirnov et al., Sci. Adv. 3, e1603179 (2017).

[29] R. Yoshimi, A. Tsukazaki, Y. Kozuka, J. Falson, K. S. Takahashi, J. G. Checkelsky, N. Nagaosa, M. Kawasaki, and Y. Tokura, Nat. Commun. 6, 6627 (2015).

[30] Y. Xu, I. Miotkowski, and Y. P. Chen, Nat. Commun. 7, 11434 (2016).

[31] M. Barkeshli and X.-L. Qi, Phys. Rev. X 4, 041035 (2014).

[32] M. R. Peterson, Y.-L. Wu, M. Cheng, M. Barkeshli, Z. Wang, and S. Das Sarma, Phys. Rev. B 92, 035103 (2015).

[33] M. Barkeshli, Phys. Rev. Lett. 117, 096803 (2016).

[34] W. Zhu, Z. Liu, F. D. M. Haldane, and D. N. Sheng, Phys. Rev. B 94, 245147 (2016).

[35] T.-S. Zeng, W. Zhu, and D. N. Sheng, Phys. Rev. B 95, 125134 (2017).

[36] D. Zhang, S. Schmult, V. Venkatachalam, W. Dietsche, A. Yacoby, K. von Klitzing, and J. Smet, Phys. Rev. B 87, 205304 (2013).

[37] S. Hennel, P. Scheidegger, M. Kellermeier, A. Hofmann, T. Krahenmann, C. Reichl, W. Wegscheider, T. Ihn, and K. Ensslin, Phys. Rev. B 97, 245305 (2018).

[38] I. P. Radu, J. B. Miller, C. M. Marcus, M. A. Kastner, L. N. Pfeiffer, and K. W. West, Science 320, 899 (2008).

[39] M. Dolev, M. Heiblum, V. Umansky, A. Stern, and D. Mahalu, Nature (London) 452, 829 (2008).

[40] X. Lin, C. Dillard, M. A. Kastner, L. N. Pfeiffer, and K. W. West, Phys. Rev. B 85, 165321 (2012).

[41] S. Baer, C. Rossler, T. Ihn, K. Ensslin, C. Reichl, and W. Wegscheider, Phys. Rev. B 90, 075403 (2014). 
[42] H. Fu, P. Wang, P. Shan, L. Xiong, L. N. Pfeiffer, K. West, M. A. Kastner, and X. Lin, Proc. Natl. Acad. Sci. U.S.A. 113, 12386 (2016).

[43] G. Yang and D. E. Feldman, Phys. Rev. B 88, 085317 (2013).

[44] X.-G. Wen, Phys. Rev. B 44, 5708 (1991).

[45] See the Supplemental Material at http://link.aps.org/ supplemental/10.1103/PhysRevLett.124.246801 for (1) the simulation of the potential profile and density in the region of the quantum point contact, (2) key transport quantities derived from the Landauer-Büttiker formalism, and (3) additional data sets of the tunnel conductance near $\nu_{\text {tot }}=1$ for two different QPCs.

[46] Based on Landauer-Büttiker formalism, the longitudinal resistance across the QPC $R_{T}$ follows: $R_{T}=R_{D}-R_{x y}$. We show in the Supplemental Material that this is indeed the case. Furthermore, $R_{T}\left(I_{\mathrm{dc}}\right)$ gives rise to essentially the same result as $R_{D}\left(I_{\mathrm{dc}}\right)$.

[47] S. Roddaro, V. Pellegrini, F. Beltram, G. Biasiol, and L. Sorba, Phys. Rev. Lett. 93, 046801 (2004).

[48] The relative error is calculated by taking the average of the absolute values of fit residuals at a fixed combination of $g$ and $e^{*} / e$. This value is further divided by $0.004 h / e^{2}-$ the noise of measurement. This measurement noise is evaluated from the resistance fluctuation in the high bias range $\left(I_{\mathrm{dc}}\right.$ from -14 to $\left.-11 \mathrm{nA}\right)$ : we take three times the standard deviation $\sigma$ in this range.

[49] T. Hyart and B. Rosenow, Phys. Rev. B 83, 155315 (2011). 